\title{
PROGRAMA DE ATENÇÃO AO IDOSO: RELATO DE UM MODELO ASSISTENCIAL ${ }^{1}$
}

\author{
Evelise Moraes Berlezi², Heloisa Meincke Eickhoff, Karla Renata de Oliveira ${ }^{4}$, Loiva Beatriz Dallepiane ${ }^{5}$, \\ Nara Marilene Oliveira Girardon Perlini ${ }^{6}$, Aline Mafalda ${ }^{7}$ Cristiane Bueno ${ }^{8}$
}

\footnotetext{
${ }^{1}$ Artigo elaborado a partir da experiência do projeto de extensão - Programa de Atenção ao Idoso: proposição de modelo assistencial, da Universidade Regional do Noroeste do Estado do Rio Grande do Sul (UNIJUí).

${ }^{2}$ Doutora em Gerontologia Biomédica. Docente do Departamento de Ciências da Saúde UNIJUÍ. Rio Grande do Sul, Brasil. E-mail: evelise@unijui.edu.br

${ }^{3}$ Mestre em Educação nas Ciências. Docente do Departamento de Ciências da Saúde da UNIJUÍ. Rio Grande do Sul, Brasil. E-mail: heloisa@unijui.edu.br

${ }^{4}$ Mestre em Ciências Biológicas: Bioquímica. Docente do Departamento de Ciências da UNIJUí. Rio Grande do Sul, Brasil. E-mail: karla@unijui.edu.br

${ }^{5}$ Doutora em Ciências da Saúde: Geriatria. Docente do Departamento de Ciências da UNIJUÍ. Rio Grande do Sul, Brasil. E-mail: loiva.dallepiane@hotmail.com

${ }^{6}$ Doutora em Enfermagem. Docente do Departamento de Ciências da UNIJUÍ. Rio Grande do Sul, Brasil. E-mail: nara.girardon@ gmail.com

7 Acadêmica do curso de Fisioterapia da UNIJUÍ. Bolsista de Extensão PIBEX/UNIJUí 2009. Rio Grande do Sul, Brasil. E-mail: aline.mafalda@hotmail.com

${ }^{8}$ Acadêmica do curso de Farmácia da UNIJUÍ. Bolsista de Extensão PIBEX/UNIJUí 2009. Rio Grande do Sul, Brasil. E-mail: cryssbueno@yahoo.com.br
}

\begin{abstract}
RESUMO: Este artigo trata do relato de um projeto de extensão universitária que propõe um modelo de atenção ao idoso. O objetivo do programa é oferecer assistência a idosos de baixa renda residentes no município de Ijuí-RS, Brasil, em situação de fragilidade e com risco de médio a alto de internação ou reinternação hospitalar. São incluídas no projeto as demandas de idosos das Unidades Básicas de Saúde, do Hospital de Caridade de Ijuí, e as dos que buscam o projeto por via telefônica. A equipe interdisciplinar, composta de professores e acadêmicos dos cursos de enfermagem, farmácia, fisioterapia e nutrição, realizam a avaliação da condição de saúde do idoso através de instrumentos quantitativos, que categorizam os indivíduos em classes de risco e elegem a prioridade de atenção. Os idosos que integram o programa recebem atendimento ambulatorial ou no domicílio, dependendo das condições de acesso e capacidade funcional.
\end{abstract}

DECRITORES: Saúde do idoso. Assistência a idosos. Internação hospitalar. Equipe de assistência ao paciente.

\section{THE ELDERLY CARE PROGRAM: REPORT OF AN ASSISTENTIAL MODEL}

\begin{abstract}
This article reports on a university extension program that proposes an elderly care model. The program's objective is to offer assistance to low-income elderly who live in a fragile situation and with medium to high risk of hospitalization or re-hospitalization in Ijuí City, Rio Grande do Sul, Brazil. The project includes the demands of the elderly from Brazilian Public Health Care Clinics and the municipal hospital (Hospital de Caridade de Ijuí), as well as those who seek the project by phone. The interdisciplinary care team, which is composed of teachers and students from Nursing, Pharmacy, Physical Therapy, and Nutrition undergraduate programs, carries out the evaluation of the health condition among the elderly involved with the use of quantitative tools to categorize the individuals in risk classes and select the priority for assistance. The elderly who are part of the program receive ambulatory or in-home care, depending on the conditions of access and functional capacity
\end{abstract}

DECRIPTORS: Health of the elderly. Old age assistance. Hospitalization. Patient care team

\section{PROGRAMA DE ATENCIÓN A ANCIANOS: INFORME DE UN MODELO DE ASISTENCIA}

RESUMEN: El artículo trata sobre el relato de un proyecto de extensión universitaria en el que se propone un modelo de atención a ancianos. El objetivo del programa es brindar asistencia a los ancianos de escasos recursos residentes en el municipio de Ijuí-RS, Brasil, en situaciones de fragilidad y con alto o medio riesgo de hospitalización o rehospitalización. Se incluyen en el proyecto las demandas de los ancianos de las unidades básicas de salud, del Hospital de Caridad de Ijuí, y los que buscan el proyecto vía telefónica. El equipo interdisciplinario, compuesto por profesores y estudiante de los cursos de enfermería, farmacia, fisioterapia y nutrición, realiza la evaluación del estado de salud de los ancianos a través de instrumentos cuantitativos, que clasifican a los individuos en clases de riesgo y luego eligen la atención prioritaria. Los ancianos que participan del programa reciben atendimiento de forma ambulatoria o en casa, dependiendo de las condiciones de acceso y capacidad funcional.

DESCRIPTORES: Salud de los ancianos. Asistencia a los ancianos. Hospitalización. Equipo de atención al paciente 


\section{INTRODUÇÃO}

O desafio maior no século XXI, no Brasil, será cuidar de uma população de mais de 32 milhões de idosos, a maioria com baixo nível socioeconômico e educacional, e com alta prevalência de doenças crônicas e incapacitantes. ${ }^{1}$

Em menos de 40 anos, o Brasil passou de um perfil de mortalidade típico de uma população jovem, para um quadro caracterizado por enfermidades complexas e onerosas, próprias das faixas etárias mais avançadas. Esse fato acarreta crescimento das despesas com tratamentos médicos e hospitalares, e, ao mesmo tempo, representa mais um desafio para as autoridades sanitárias, especialmente no que tange à implantação de novos modelos e métodos e planejamento, gerência e prestação de cuidados. ${ }^{2}$

O hospital, por sua vez, ainda é o espaço hegemônico de formação dos profissionais de saúde. Essa presença marca as competências dos profissionais, mesmo quando se encontram trabalhando na Atenção Básica. ${ }^{3-4}$

Os critérios de intervenção e a alta de um hospital acabam definindo objetivos dos profissionais e, consequentemente, o tempo de contato da equipe com o doente. Esses critérios e tempos produzem expectativas, prioridades e certa sensação de eficácia. O Pronto-Socorro (PS) evidencia com seus limites o que é isso. O PS é capaz de atender infinitas vezes um hipertenso em crise, sem fazer intervenções para evitar a recorrência, e manter, ainda assim, a sensação de eficácia. Uma equipe, na Atenção Básica, que levasse seis meses para ajudar um paciente com hipertensão grave, há cinco anos, a controlar sua pressão arterial poderia considerar-se muito eficaz. São, portanto, tempos diferentes, mas a formação excessivamente intrahospitalar é intrinsecamente imediatista e não possibilita o aprendizado de um raciocínio clínico e a realização de projetos terapêuticos de médio e de longo prazo. ${ }^{3}$

Em 2001, o Sistema de Internação Hospitalar (SIH) do Sistema Único de Saúde (SUS) registrou 12.227.465 internações hospitalares no âmbito do SUS. Os idosos, que representavam $8,5 \%$ da população geral, responderam por $18,3 \%$ das hospitalizações. A razão de habitantes/internações aumentou acentuadamente com a idade: 1,0; 1,7; 2,4 e 3,4 nas faixas etárias de 20-59, 60-69, 70-79 e $80+$ anos de idade, respectivamente. ${ }^{5}$

O sistema de saúde não está estruturado para atender à demanda crescente desse segmen- to etário. Os idosos consomem mais dos serviços de saúde, suas taxas de internação são bem mais elevadas e o tempo médio de ocupação do leito é muito maior quando comparados a qualquer outro grupo etário. A falta de serviços domiciliares e/ou ambulatoriais faz com que o primeiro atendimento ocorra em estágio avançado, no hospital, aumentando os custos e diminuindo as chances de prognóstico favorável. Em outras palavras, consomem-se mais recursos do que seria preciso, elevam-se os custos, sem que, necessariamente, se obtenham os resultados esperados em termos de recuperação da saúde e melhoria da qualidade de vida. ${ }^{6}$

Determinar o valor de uma intervenção de assistência à saúde requer que façamos uma avaliação da qualidade e do custo de tratamento. A qualidade de um procedimento é especialmente medida pela sua capacidade de produzir consequências desejáveis ao paciente. As consequências monitoradas pela maior parte dos estudos voltados à verificação da eficácia da Avaliação Geriátrica Completa (AGC) são o índice de mortalidade, a readmissão hospitalar, os índices de institucionalização e o nível das funções físicas e cognitivas do paciente. ${ }^{7}$

O idoso apresenta peculiaridades distintas das demais faixas etárias e, por este motivo, sua avaliação de saúde deve ser feita objetivando a identificação de problemas subjacentes à queixa principal, incluindo as avaliações funcionais, cognitivas, psíquicas, nutricionais e sociais, que interferem diretamente na sua saúde e no grau de sua autonomia e independência. A falta de uma avaliação geriátrica correta está diretamente ligada à reinternação e ao maior uso de serviços de saúde observados. ${ }^{8}$

Com relação aos gastos de saúde, os dados do SUS mostram que eles são proporcionalmente muito mais altos entre a população idosa do que entre a população de zero a 14 anos, por exemplo. Qual é o custo proporcional da hospitalização das pessoas idosas? Muitas vezes, essa hospitalização poderia ser evitada; outras vezes os idosos ficam longo tempo internados no hospital. A assistência domiciliar vem para desospitalizar precocemente, mas não inadequadamente: o idoso só sai do hospital quando está suficientemente estável para ser acompanhado em casa. Isso repercute de forma importante nos custos de assistência à saúde. ${ }^{9}$

Dados do SUS mostram que a população idosa, em 1996, representou 7,3\% das autorizações de internação, consumindo $22,9 \%$ dos recursos 
gastos. O índice de custo hospitalar (custo de hospitalização consumido por habitante/ano) foi de $\mathrm{R} \$ 10,54$ para o segmento de $0-14$ anos, $\mathrm{R} \$$ 16,20 para o de $15-59$ anos e $R \$ 59,40$ para o de 60 anos ou mais. O tempo de permanência estimado foi de 5,3 dias para 0-14 anos, 5,1 dias para 15-59 anos e 7 dias para 60 anos ou mais. O índice de hospitalização (número de dias de hospitalização usado por habitante/ano) foi de 0,24 dias para 0-14 anos, 0,38 dias para 15-59 anos e 1,27 dias para 60 anos ou mais. Portanto, com o esperado aumento nessa parcela da população, pode-se inferir que os gastos com saúde serão também progressivamente maiores. ${ }^{8}$

No entanto, grandes gastos não garantem uma efetividade das ações. Ou seja, um trabalho voltado para uma melhoria real da qualidade de vida dos idosos requer muito mais do que investimentos em tecnologia hospitalar. O controle dos gastos com o tratamento dos idosos passa, em primeiro lugar, pelo investimento nas formas de promoção da saúde, que se traduzem por qualidade de vida e não por tratamento de doenças. Diante desse cenário, o setor saúde ainda busca meios eficazes que possam equacionar adequadamente os problemas que acompanham o processo de envelhecimento. Uma das formas de alcançar sucesso nesse desafio está relacionada à construção de novos modelos de atenção que fujam à prática hospitalocêntrica e avancem para a identificação precoce de problemas que possam ser tratados no âmbito ambulatorial. ${ }^{10}$

É necessário, portanto, formular novas concepções de assistência à saúde da população idosa, que consigam englobar as diferentes condições de saúde desse segmento etário, respeitando suas características especiais e peculiares. Os clássicos modelos de promoção, prevenção, assistência e reabilitação não podem ser mecanicamente transportados para grupos de indivíduos idosos, sem que algumas adaptações importantes e significativas sejam realizadas. O conhecimento do perfil de hospitalização dessa população é importante para o planejamento das ações de saúde. ${ }^{5}$

Não se pode esquecer, também, do impacto da hospitalização na funcionalidade do idoso. Em geral, ele sai com sua funcionalidade comprometida, não pela doença que o levou à internação, mas pelo próprio processo de hospitalização. ${ }^{9} \mathrm{O}$ declínio das atividades básicas (tomar banho, vestir-se, usar o banheiro, transferir-se da cama para a cadeira, controle esfincteriano e alimentar-se sozinho) e instrumentais de vida diária (usar o telefone, fazer compras, preparar o próprio alimento, cuidar da casa, usar o transporte público, tomar os remédios e manusear o dinheiro) pode ocorrer em um terço dos idosos hospitalizados, após a alta hospitalar, independentemente do motivo da internação. A perda funcional está intimamente associada à perda da qualidade de vida, re-hospitalização, institucionalização e óbito. ${ }^{11}$

Os determinantes da perda da independência funcional em idosos hospitalizados são múltiplos e dependem basicamente das seguintes variáveis: reserva funcional prévia, diagnóstico etiológico, tratamento instituído e os efeitos do descondicionamento físico. Os diagnósticos mais comumente associados às principais incapacidades são o acidente vascular cerebral, fratura de fêmur, insuficiência cardíaca congestiva, pneumonia, insuficiência coronariana e câncer. Os principais fatores de risco são: idade maior de 75 anos, baixa performance no exame do estado mental, dependência prévia em duas ou mais atividades instrumentais de vida diária e baixo nível de atividade social. A consequência da internação entre os efeitos do envelhecimento e da hospitalização é a "cascata da dependência", culminando, por vezes, na institucionalização definitiva do idoso. ${ }^{11}$

Assim, nesta perspectiva, foi idealizado um projeto de extensão intitulado Programa de Atenção ao Idoso (PAI): proposição de um modelo assistencial, pois a realidade do município de Ijuí-RS, onde o projeto é desenvolvido, evidencia a necessidade de ações em saúde para os idosos em função de não existir uma política de saúde específica e, também, pela casuística de idosos fragilizados necessitando de assistência direta em seu domicílio.

Neste contexto teórico e da realidade local e assumindo o compromisso de uma formação acadêmica voltada para a mudança social, o Departamento de Ciências da Saúde da Universidade Regional do Noroeste do Estado do Rio Grande do Sul, através dos cursos de Enfermagem, Farmácia, Fisioterapia e Nutrição, implantou o projeto. O modelo construído a partir de uma lógica própria, que compreende atividades organizadas num fluxo hierarquizado com atividades de promoção da saúde, prevenção, acompanhamento e tratamento de doenças e referenciamento para centros de avaliação e reabilitação geriátricos. Tal modelo incorpora tecnologias atuais em relação à identificação, avaliação e tratamento de idosos, refletindo mudanças no paradigma do cuidado à população idosa. ${ }^{2}$ 
DESCRIÇÃO DO PROGRAMA DE ATENÇÃO AO IDOSO (PAI)

O PAI foi construído a partir de reflexões acadêmicas relacionadas ao cuidado à pessoa no processo de envelhecimento, entendendo ser necessária uma atenção interdisciplinar fundamentada em um modelo que assegure assistência integral ao idoso. A partir dessas concepções, foi proposto o PAI, que se caracteriza como um projeto de extensão, reconhecido institucionalmente, e desenvolvido pelos cursos de graduação de Enfermagem, Fisioterapia, Farmácia e Nutrição.

O projeto adotou um modelo que tem como princípios a organização da demanda, a partir da estratificação de risco de reinternações hospitalares e ações em níveis crescentes de complexidade, que viabilizem a progressão na estrutura da atenção. ${ }^{12}$ Destaca-se que o referencial utilizado para a construção do modelo do PAI tem sua experiência pautada na atenção ambulatorial, enquanto o PAI propõe um modelo de atenção ambulatorial e domiciliar.

O PAI encontra-se localizado na estrutura física da Unijuí Saúde, espaço acadêmico onde se desenvolvem atividades pedagógicas ambulatoriais de assistência à saúde dos cursos de graduação da área da saúde da Unijuí.

O objetivo do Programa é desenvolver um modelo de assistência a idosos de baixa renda que residam na área urbana do município de Ijuí-RS, em situação de fragilidade, e com risco de médio a alto de internação/reinternação hospitalar. Visa, ainda, potencializar e incentivar a discussão e a criação de políticas públicas de atenção ao idoso no município de ljuí, entendendo-se que a rede de atenção básica deve ser capaz de identificar e assistir, de forma eficiente, idosos fragilizados, isto é, aqueles com maior risco de desenvolver incapacidade funcional e de acompanhar os de menor risco, paralelamente ao desenvolvimento de ações e atividades de educação e promoção de saúde.

Em relação às ações desenvolvidas pelo PAI existe o propósito de melhorar a condição de saúde do idoso e reduzir o risco de internação/reinternação hospitalar através da assistência contínua, integral e interdisciplinar.

\section{A ESTRUTURA DO PAI}

A proposta deste programa inclui a participação de uma equipe interdisciplinar, cujos integrantes estão devidamente capacitados para nele atuar. Estruturalmente, o PAI possui a etapa de captação do idoso, de triagem rápida, de classificação do risco e da inclusão na assistência e reabilitação. A inserção do idoso neste programa foi pensada de forma hierarquizada (Figura 1), uma vez que as etapas se diferenciam pela profundidade e abrangência das ações, organizadas em níveis crescentes de complexidade, que permitem selecionar subgrupos de indivíduos que, por suas características de risco, devem progredir, diferenciadamente, na estrutura de atenção. ${ }^{12}$

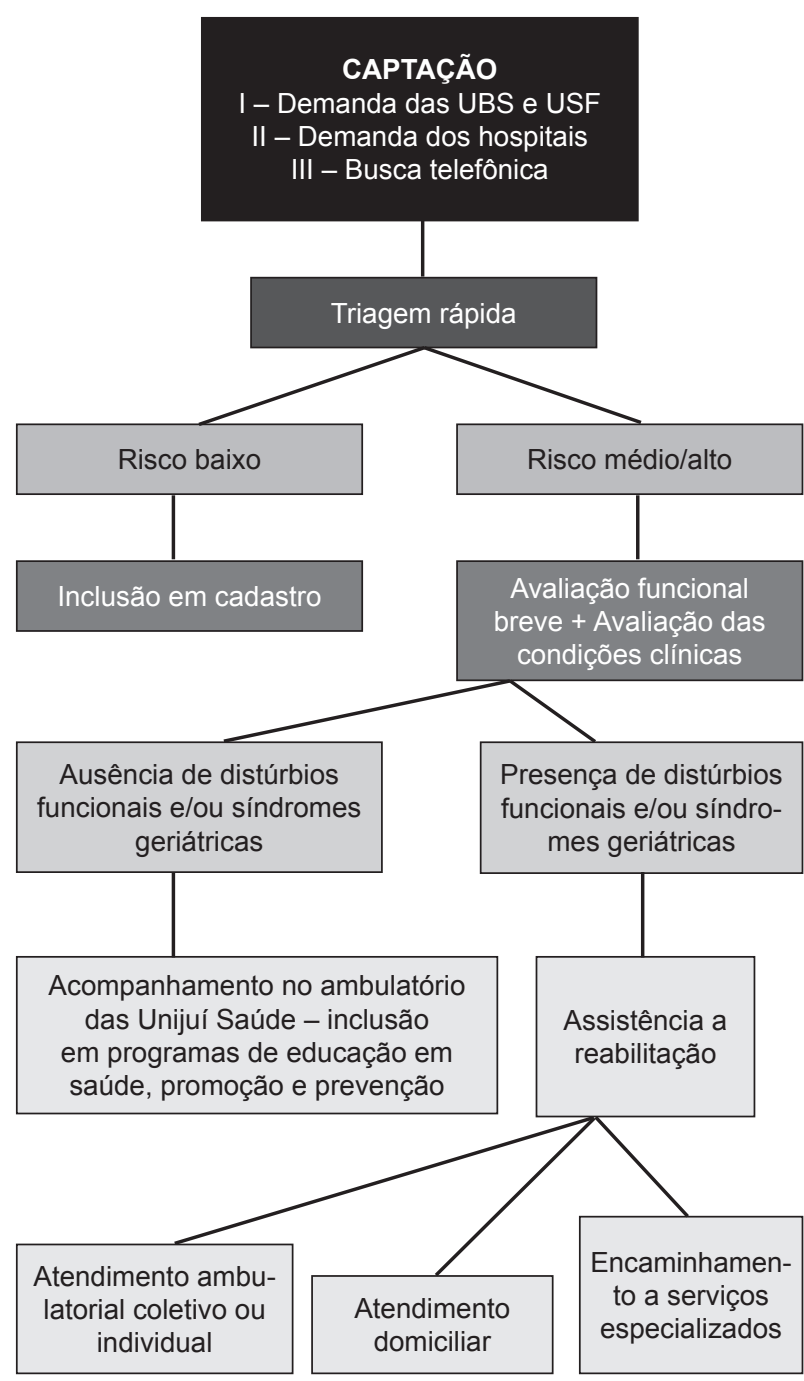

Figura 1 - Diagrama do Programa de Atenção ao Idoso (PAI)

Fonte: Adaptado de Lourenço et al. ${ }^{12}$

\section{CAPTAÇÃO E AVALIAÇÃO DO IDOSO}

De acordo com o modelo proposto, o idoso pode ser inserido no programa de três maneiras: pela demanda das unidades básicas de saúde, demanda dos hospitais e por busca telefônica (contato direto dos familiares). Os critérios de inclusão consideram a idade do idoso, que deverá ter mais de 60 
anos, baixo nível socioeconômico e risco de internação/reinternação hospitalar de médio a alto.

Após o agendamento do idoso, a equipe do PAI realiza, no primeiro momento/contato, a avaliação de todos os critérios de inclusão e do risco de internação hospitalar, utilizando o instrumento de identificação denominado de Triagem Rápida de Risco. ${ }^{13}$ Esse instrumento é de aplicação rápida, categoriza os indivíduos avaliados em classes de risco, permite organizar, por meio de critérios objetivos, diferentes níveis de intervenção, possibilita a determinação de prioridades na atenção, é de fácil padronização e compreensão no treinamento dos entrevistadores e pode ser aplicado face-a-face com o entrevistado, por telefone ou por carta.

Esse instrumento é capaz de identificar, por meio de uma fórmula de regressão logística que classifica o idoso de maior ou menor risco, indivíduos com doenças graves ou em risco de desenvolvê-las. Os critérios avaliados são: idade, sexo, disponibilidade de cuidador, autopercepção da saúde, presença de doença cardíaca, presença de diabetes mellitus, pernoite hospitalar e consultas médicas nos últimos 12 meses. A classificação do risco está descrita na tabela 1.

\section{Tabela 1 - Estratos de risco de fragilização}

\begin{tabular}{lcc}
\hline Nível & Prh $^{*}$ & Estratificação de risco \\
\hline Risco 0 & $<0,300$ & Baixo \\
Risco 1 & 0,300 a 0,399 & Médio \\
Risco 2 & 0,400 a 0,499 & Médio/alto \\
Risco 3 & $\geq$ a 0,500 & Alto \\
\hline
\end{tabular}

${ }^{*}$ Prh $=$ probabilidade de reinternação hospitalar. Fonte: Boult et al. ${ }^{13}$

Nesta proposta, assume-se o pressuposto básico desses autores de que, ao estimar o risco de internação repetida de uma determinada população, avalia-se a presença de um perfil de fragilização que, além de aumentar a probabilidade de internações repetidas, leva à redução progressiva da capacidade funcional e, consequentemente, à maior demanda por serviços de saúde em todos os níveis.

Ao atender aos critérios de inclusão, o idoso passa a ser assistido pelo PAI. Posteriormente, realiza-se a Avaliação Funcional Breve (AFB), cujo objetivo é obter a condição de limitação e conhecer os problemas principais do idoso. A AFB é um instrumento composto por 11 itens, cada um avaliando uma área específica: visão, audição, função dos membros superiores e inferiores, continência urinária, nutrição, estado mental, distúrbios do afeto, atividades de vida diária, ambiente no domicílio e rede de suporte social. Trata-se de um conjunto de questões e testes de desempenho que, avaliados em outras realidades, mostraram-se altamente sensíveis à detecção de distúrbios nas áreas pertinentes. ${ }^{14-15}$

A partir dos resultados obtidos, aplicam-se os protocolos de avaliações específicos, indicados conforme o caso, tais como o Mini-Exame do Estado Mental (MEEM), a Escala de Depressão Geriátrica (GDS), a Medida de Independência Funcional (MIF), a Avaliação do Equilíbrio e Marcha de Tinetti, e o APGAR da família. ${ }^{16}$

Quanto aos idosos classificados como de baixo risco, esses passam apenas a fazer parte do cadastro do PAI. Cabe salientar que esta etapa, assim como as demais, é realizada por todos os estudantes e docentes participantes do Programa, em sistema de rodízio.

\section{A ORGANIZAÇÃO DO CUIDADO E A ATUAÇÃO DA EQUIPE INTERDIS- CIPLINAR}

Após a inclusão do idoso e a definição da sua condição de saúde, o acadêmico responsável apresenta o caso ao grupo nas reuniões agendadas. A equipe discute a avaliação e produz um plano de atenção terapêutica de acordo com as necessidades, o qual passa então a ser implementado a partir das especificidades de cada área e núcleo profissional. O plano terapêutico desenvolvido e as evoluções da assistência ao idoso são registrados nos prontuários dos pacientes pelos acadêmicos envolvidos no Projeto. Esses prontuários encontram-se arquivados na secretaria do PAI, na Unijuí Saúde.

A Fisioterapia atua na reabilitação do idoso e prioriza a realização de técnicas visando à manutenção, melhora ou restauração da sua capacidade funcional, bem como a monitorização dessa condição. Ainda, é função da Fisioterapia a adaptação de utensílios e espaços domésticos para que o idoso consiga desempenhar suas atividades de vida diária com a máxima independência. Na busca pela otimização do cuidado e reabilitação, também é realizado um trabalho de suporte ao cuidador familiar, com ênfase na informação para que ele possa agir adequadamente nos posicionamentos, no manejo das trocas de decúbitos, nos cuidados para prevenção de escaras, no incentivo às atividades funcionais e em outras necessidades impostas pela realidade vivenciada em cada família. 
A Nutrição realiza, após a triagem de idoso em risco nutricional, uma avaliação nutricional e dietética específica, a fim de identificar os problemas nutricionais, definir objetivos e metas e propor um plano terapêutico baseado nas condições clínicas, físicas, socioeconômicas e culturais do paciente e do núcleo familiar, sugerindo as adaptações necessárias para melhorar o consumo alimentar. Considerando o envelhecimento como um processo, a nutrição tem, ainda, como objetivo, trabalhar em consonância com a equipe, compreendendo a multideterminação dos problemas de saúde vivenciados pelos idosos, apoiados no conceito da interdisciplinaridade.

A contribuição da Farmácia no PAI se dá através do acompanhamento da terapia medicamentosa instituída, considerando a resposta individual dos usuários ao tratamento. Procura-se verificar a possibilidade de não adesão ao tratamento e identificar suas possíveis causas para, então, estabelecer estratégias de adesão. A partir disso, procede-se à orientação do usuário ou seu cuidador sobre o uso adequado dos medicamentos. A identificação de possíveis interações medicamentosas e interferências do horário da administração dos medicamentos no trabalho das demais áreas da saúde também são consideradas.

A Enfermagem considera o idoso integrante do sistema familiar. Por isso, procura, a partir das evidências identificadas na avaliação funcional e clínica do idoso e do contexto de vida da família, utilizar a Sistematização da Assistência da Enfermagem como instrumento norteador da prática, incluindo, além dos aspectos físicos e psicossociais, o genograma e o ecomapa da família, como instrumentos que favorecem a identificação das necessidades atuais do idoso para, então, realizar o planejamento das estratégias de intervenção na situação. Com base nesses elementos, procura desenvolver um trabalho compartilhado com os demais integrantes da equipe, com o idoso e a família, no sentido de atender, de modo integral, as necessidades evidenciadas, bem como as demandas apresentadas espontaneamente pelo idoso ou familiares. Para tanto, promove e propõe ações de prevenção de agravos e de tratamento às situações já instaladas, considerando o campo profissional. Estimula a autonomia e o autocuidado pelo idoso, conforme o grau de dependência. Incentiva, orienta e ensina à família e/ou cuidadores informais ações e técnicas para o desenvolvimento de habilidades motoras, assunção e desempenho de cuidados necessários e que o idoso não apresente condições de realizar, enfatizando os aspectos positivos de cada pessoa com quem interage neste processo. Desse modo, a Enfermagem, além de propor e implementar ações específicas de cuidado, conforme a necessidade e o grau de fragilidade do idoso, procura potencializar as forças existentes na unidade familiar.

Ainda, são organizados seminários sobre assuntos que permeiam a realidade do Programa, a fim de qualificar teoricamente o cuidado prestado ao idoso e sua família e embasar a assistência que é proporcionada. Assim, ocorre permanentemente a qualificação e a formação continuada da equipe.

Todas as áreas trabalham de acordo com os propósitos que fundamentam o Programa, efetivando reavaliações periódicas para a identificação do risco de internação ou reinternação e necessidade de reprogramação do plano terapêutico, com vistas a incentivar o autocuidado, a independência funcional e a autonomia do idoso.

Para que um idoso integre o projeto são respeitados os aspectos éticos das relações profissionais. A equipe do PAI construiu, junto ao Hospital de Caridade de Ijuí (HCI), a logística para que todo idoso, ao internar, tenha em seus documentos de admissão hospitalar um formulário de autorização do médico responsável, para que a equipe do PAI possa a ter acesso ao idoso e avaliá-lo. Diariamente há, no HCI, acadêmicos sob supervisão docente, que percorrem as unidades de internação e verificam, junto à equipe de Enfermagem, internações de idosos. Havendo nova internação, o acadêmico desloca-se até o leito, identifica-se ao idoso e/ou familiar e, no primeiro momento, apresenta o projeto. Se houver o aceite, por parte do próprio idoso ou responsável, em integrar o projeto, é realizada a avaliação do risco de internação/reinternação hospitalar, seguindo o protocolo proposto, quando o risco for de médio a alto.

Quando o idoso está domiciliado e a busca pelo projeto é por via telefônica, a equipe do PAI, com conhecimento da localização da residência, contata com a equipe da Unidade Básica de Saúde (UBS) de referência, para não haver trabalhos paralelos entre a equipe do PAI e da referida Unidade.

$\mathrm{Na}$ atuação da equipe, pode haver a necessidade do atendimento de todas ou de algumas áreas (Fisioterapia, Nutrição, Farmácia e Enfermagem). Isso significa que a condição do paciente é a orientadora da necessidade da atenção e da demanda de tempo e terapêuticas desenvolvidas. Assim, otimiza-se a atuação do Programa e mantém-se um trabalho integrado entre os estudantes. 
Os idosos com risco médio a alto e ausência de distúrbios funcionais e/ou síndromes geriátricas são atendidos no espaço da Unijuí Saúde, mediante agendamento. Isso porque são pacientes que apresentam condições de se deslocarem. Já aqueles com a presença de distúrbios funcionais e/ou síndromes geriátricas, que apresentam incapacidades funcionais e/ ou dificuldades de acesso a transporte, são atendidos no seu domicílio, respeitada a dinâmica de atendimento das UBS onde o usuário está cadastrado (Figura 1). Além disso, o Programa procura estabelecer uma relação de cooperação e parceria com as Unidades Básicas de Saúde do município.

\section{AIMPORTÂNCIADOPAINA FORMAÇÃO DO PROFISSIONAL DA SAÚDE}

O PAI, para além de se apresentar como um modelo assistencial que visa atender a uma demanda imposta pela realidade do envelhecimento populacional brasileiro, traz importantes contribuições na formação do profissional da saúde. Isso porque é necessário que se acompanhe a lógica da realidade, que exige do profissional da saúde conhecimento para atuar nas necessidades que o envelhecimento acarreta e, também, uma disponibilidade para atuar de forma interdisciplinar.

O trabalho em equipe propicia na formação do profissional de saúde o desafio da construção de saberes para e com a pessoa cuidada. O conceito de cuidado compartilhado amparado nos pressupostos do construcionismo social, entendendo que o conhecimento se constrói a partir da experiência do sujeito em um processo de significação. Este conceito de cuidado abrange uma dimensão educativa que implica em mobilização de saberes e afetos, importantes para uma prática solidária e ética, para que possa ser verdadeiramente compartilhada. ${ }^{17}$

A mudança da pirâmide etária e do perfil epidemiológico brasileiro impõe aos educadores / formadores o compromisso de formar profissionais de saúde voltados para as necessidades de uma população de idade mais avançada, que apresenta maior prevalência de doenças, demanda mais serviços de saúde e de maior complexidade e, acima de tudo, demanda maior cuidado. Contudo, a maioria das instituições brasileiras de ensino da área de saúde ainda não despertou para o atual processo de transição demográfica e epidemiológica e suas consequências médico-sociais, não oferecendo conteúdo gerontológico adequado em seus cursos de graduação. Com isso, amplia-se a carência de recursos técnicos e humanos para enfrentar a explosão desse grupo populacional nas próximas décadas. Nesse sentido, os cursos da área da saúde têm o compromisso de inserir a temática do envelhecimento, seja como componente curricular elencado no Projeto Pedagógico do Curso, seja permeando a formação teórico-prática nos cursos.

\section{CONCLUSÕES}

O PAI se constitui em um projeto de extensão universitária que contempla um modelo de assistência ao idoso de forma a estimular novas práticas de atenção à saúde para essa faixa etária, que apresenta condições e necessidades diferenciadas, assim como se constitui numa prática que potencializa a formação acadêmica interdisciplinar de profissionais da saúde.

Este relato permite refletir sobre a importância das atividades de extensão como elemento agregador na formação do estudante de saúde, constituindo-se num caminho teórico e metodológico na perspectiva de romper com a prática fragmentada do cuidado à saúde, apostando na integralidade mediada pela interdisciplinaridade.

O PAI, ao propiciar a vivência interdisciplinar, possibilita o estabelecimento de um espaço onde os futuros profissionais da saúde exercitam o diálogo, tenham momentos de troca de conhecimentos técnico-científicos, construam novos saberes na prática de atenção geriátrica e reflitam sobre o seu fazer, beneficiando e qualificando a intervenção terapêutica nos sujeitos/pacientes que estão sob seus cuidados. Entendendo que a formação de recursos humanos em geriatria e gerontologia é o primeiro passo para qualificar a atenção ao idoso, esta formação é especialmente importante para as demandas que o sistema de saúde brasileiro terá de enfrentar com o envelhecimento populacional.

A experiência vivenciada a partir deste projeto de Extensão universitária permite inferir que se trata de modelo viável a ser adotado pelo município, no qual o Programa é desenvolvido, bem como servir de proposta para outros. Avalia-se, qualitativamente, que as ações do PAI são efetivas na resolutividade de grande parte dos problemas de saúde dos idosos atendidos, especialmente em relação à redução do risco de reinternação hospitalar. Além disso, constituem-se em atividades e procedimentos que demandam baixos investimentos pelos municípios. 
Contudo, o grande desafio da implantação deste modelo ou semelhantes está na capacidade da equipe de saúde de atuar de forma interdisciplinar e na integralidade da atenção ao idoso. Para a efetividade desta proposta, o planejamento de todas as ações, a discussão individualizada dos casos e o acompanhamento pela equipe são fundamentais. De qualquer forma, vale ressaltar que se aposta-se nesta proposição de modelo como nova prática em saúde na comunidade local, possibilitando que ela seja disseminada no contexto locorregional e pelos gestores que se encontram à frente da organização e implementação de políticas públicas.

\section{REFERÊNCIAS}

1. Ramos LR. A mudança de paradigma na saúde e o conceito de capacidade funcional. In: Ramos LR, Neto JT, coordenadores. Guias de geriatria e gerontologia. (Guias de medicina ambulatorial e hospitalar). Barueri (SP): Manole; 2005. p.1-7.

2. Veras R. Em busca de uma assistência adequada à saúde do idoso: revisão da literatura e aplicação de um instrumento de detecção precoce e de previsibilidade de agravos. Cad Saúde Pública. 2003 Mai-Jun; 19(3):705-15.

3. Cunha GT. A construção da clínica ampliada na atenção básica. São Paulo (SP): Hucitec; 2005.

4. Carvalho SR. Saúde coletiva e promoção da saúde: sujeito e mudança. São Paulo (SP): Hucitec; 2005.

5. Loyola Filho AI, Matos DL, Giatti L, Afradique ME, Peixoto SV, Lima-Costa MF. Causas de internações hospitalares entre idosos brasileiros no âmbito do Sistema Único de Saúde. Epidemiol. Serv Saúde. 2004 Dez; 13(4):229-38.

6. Caldas CP. Fatores de risco em envelhecimento - o idoso frágil e as síndromes geriátricas. In: Saldanha AL. Saúde do idoso: a arte de cuidar. $2^{\mathrm{a}}$ ed. Rio de janeiro (RJ): Interciência; 2004. p.159-61.

7. Levy, SM. Avaliação multidimensional do paciente idoso. In: Reichel, W, Gallo JJ, Busby-Whitehead J, Rabins PV, Silliman RA, Murphy JB. Assistência ao idoso: aspectos clínicos do envelhecimento. $5^{\mathrm{a}}$ ed, Rio de Janeiro (SP): Guanabara Koogan; 2001. p.15-29.

8. Motta LB. Levantamento do perfil de idosos internados em um hospital geral: análise do processo de internação frente às demandas da população geriátrica. Textos Envelhecimento. 2001; 3(6):47-77.

9. Cucurella E, Duarte YAO. Moradias, cuidadores e atendimento domiciliar. In: Anais do Encontro Internacional de Gerontologia Social. São Paulo (SP): SESC; 2006.

10. Veras RP, Caldas CP, Coelho FD, Sanches MA. Promovendo a saúde e prevenindo a dependência: identificando indicadores de fragilidade em idosos independentes. Rev Bras Geriat. Gerontol. 2007; 10(3):355-70.

11. Moraes EN, Bandeira EMFS, Silva DF, Medeiros MCAF. Rede assistencial de atenção à pessoa idosa. In: Moraes EN. Princípios básicos de geriatria e gerontologia. Belo Horizonte (MG): Coopmed; 2008. p.665-78.

12. Lourenço RA, Martins CSF, Sanchez MAS, Veras RP. Assistência ambulatorial geriátrica: hierarquização da demanda. Rev Saúde Pública [online]. 2005 [acesso 2008 Mai 28]; 39(2). Disponível em: http:/ / scielo.br/pdf/rsp/v39n2/24058.pdf.

13. Boult C, Dowd B, McCaffrey D, Boult L, Hernandez R, Krulewitch H. Screening elders for risk of hospital admission. J Am Geriatr Soc. 1993 Aug; 41(8):811-7.

14. Lachs MS, Feinstein AR, Cooney LM, Drickamer MA, Marottoli RA, Pannill FC, et al. A simple procedure for general screening for functional disability in elderly patients. Ann Intern Med. 1990 May; 112(9):699-706.

15. Lourenço RA, Martins CSF. Cognitive impairment in the elderly people: preliminary results from Quick Functional Assessment (QFA) and Mini-Mental State Examination (MMSE). In: Joint International Meeting Psychogeriatric Association and Brazilian Association of Geriatric Neuropsychiatry \& IV Brazilian Forum of Geriatric Neuropsychiatry; 2000; Porto Alegre (RS), Brazil.

16. Ministério da Saúde (BR), Secretaria de Atenção à Saúde, Departamento de Atenção Básica. Envelhecimento e saúde da pessoa idosa. Brasília (DF): MS; 2006.

17. Teixeira MLO, Ferreira MA. Cuidado compartilhado: uma perspectiva de cuidar do idoso fundamentada na educação em saúde. Texto Contexto Enferm. 2009 Out-Dez; 18(4):750-8. 